\section{ACCIDENTAL POISONING BY IODINE.}

\section{To the Editor of THE LANCET.}

Sir,-Y Yon will oblige me by giving the following case insertion in your valuable periodical. I am, yours, \&c.

Leonard BuCKell, M.R.C.S. Chichester, Feb. 4, 1843.

Mrs. A., atat. 36, married, and having one child; is of light complexion, and nervous temperament, generally enjoying good, though not robust, health. She has a tumour situated opposite and over the sixth and seventh cervical, and first and second dorsal vertebræ, that has been five years attaining its present size, which is about that of a hen's egg, and has lately been treated by applications of nitrate of silver, with evident diminution of the swelling, and decrease in the pain which has always attended it. The nitrate of silver was applied for the last time on the 22nd $\boldsymbol{J}$ anuary.

23. Eleven, a.m. The caustic has excoriated the surface, but the tumour remains unaltered. Ordered the external use of the tincture of iodine, diluted with half its own measure of spirits of wine. When this application came in contact with the excoriated portion of the swelling the patient instantly experienced violent pain, and a sense of weight in the epigastric region, attended with universal tremors, faintness, cold and profuse perspirations, extreme collapse of the countenance, involuntary twistings of the arms, particularly the right, chattering of the teeth, inability to stand, and a pulse small, weak, and slow. To this condition were quickly added violent vomiting and spasm. The patient was with difficulty renroved to bed, when some gin and water were administered, and a hot fomentation was ordered to the epigastric region, after which the following draught was given every two hours :-

R Compound tinctures of cinchona bark and cardamoms, of each three drachms;

Comp. spirit of ammonia, a drachm and a half ;

Peppermint water, an ounce and a half. Mix for a draught.

Seven, p.m. Pain and weight at epigastrium considerably relieved by the treatment. General pain, however, much increased, and vomiting is induced by assuming the erect position. Urine is continually dribbling away, attended with a constant desire of voiding it; feet cold; pulse weak and tremulous; bowels constipated. Apply bottles of hot water to the feet, and give a tablespoonful of castor oil every three hours until the bowels act freely.

24. Eleven, a.m. All the distressing symptoms are much relieved, though still aggravated upon rising in bed. Urine can be retained for an hour or more. Menstrual discharge has commenced, though not very profusely ; the patient had been unwell eight days previously; bowels freely opened by the castor oil. Continue medicaments.

25. The patient considerably better; but suffering now from extreme debility, with a pallid face and a tremulous pulse; appetite very bad; bowels confined. Repeat the castor oil.

30. The patient has now recovered her accustomed strength and health; the cata. menia had ceased on the day before; tumour very considerably lessened.

Here is a case in which the absorption of a very small portion of iodine into the system produced symptoms of an alarming character, dependent perhaps, not so much on the quantity of iodine entering the system, as upon some peculiarity in the constitution and condition of the individual upon whom it was used, since not more than four or five drops of the tincture had been applied before the above symptoms were produced.

\section{CARCINOMA OF THE UTERUS UN . ATTENDED BY LOCAL PAIN.}

A French practitioner has reported an instance of this kind as having taken place in a woman of forty-five years of age, who had had three children, delivery in each case having been effected without accident. Her general health appeared to be good, till one day, during a walk, she experienced a sudden and alarming uterine hæmorrhage. This was fortunately subdued; but for two months succeeding the patient was frequently in an ailing condition, though without absolute pain; and hæmorrhagic discharges from time to time occurred. At length the skin assumed a jaundiced hue, and after four days of violent pains in the lower extremities paralysis of these limbs supervened, bluish patches were perceived around the ankles, the functions of the body became much disturbed, and hectic fever ensued. The pulse fell, the surface became cold; gangrenous spots covered the upper extremities, and the patient rapidly sank. The uterus after death was found enlarged to ten times its natural size, and weighed considerably more than two pounds. Its outer surface exhibited no unusual appearance, and its muscular structure was healthy; but its mucous membrane was everywhere covered with small hardened bodies agglomerated together, and presenting a scirrhous aspect. No mechanical pressure seemed to have been exerted by the organ on the iliac arteries or veins, or on the sacral plexus of nerves, sufficient to account for the pains felt in the extremities, which must accordingly be attributed to some nervous sympathy.Gaz, des Hop. 